\title{
Areca nut extract protects against ovariectomy-induced osteoporosis in mice
}

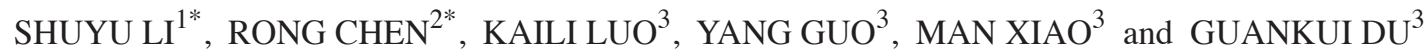 \\ ${ }^{1}$ Clinical College of Hainan Medical College, Haikou, Hainan 571199; ${ }^{2}$ Department of Orthopedics, \\ Hainan Agribusiness Central Hospital, Haikou, Hainan 570311; ${ }^{3}$ Department of Biochemistry \\ and Molecular Biology, Hainan Medical College, Haikou, Hainan 571199, P.R. China
}

Received October 20, 2015; Accepted February 10, 2017

DOI: $10.3892 /$ etm.2017.4362

\begin{abstract}
Estrogen deficiency increases the generation of reactive oxygen species (ROS), which is a crucial pathogenic factor for osteoporosis. Areca nuts are rich in phenolics, which have high antioxidant activity. In the present study, an ovariectomy (OVX)-induced osteoporosis mouse model was used to investigate the protective effects of areca nut extract (ANE) on bone loss and related processes. A total of 248 -week-old female mice were randomly divided into three groups ( $n=8$ per group): I Sham-operated control; II, bilateral OVX; and III, bilateral OVX + ANE. Group III were treated orally with ANE at a single dose of $300 \mathrm{mg} / \mathrm{kg}$ body weight daily for 6 months. ANE supplementation for 6 months improved trabecular bone microarchitecture and significantly increased bone mineral density in the distal femur $(\mathrm{P}<0.05)$ compared with Group II. Furthermore, serum levels of the osteoclast differentiation-inducing factors, receptor activator of nuclear factor $-\kappa \mathrm{B}$ ligand and osteoprotegerin were significantly increased and decreased, respectively (both $\mathrm{P}<0.05$ ), in OVX mice and these effects were significantly inhibited by ANE treatment (both $\mathrm{P}<0.05$ ). ANE supplementation also resulted in significantly decreased serum hydrogen peroxide and malondialdehyde levels compared with Group II, while the levels of glutathione and catalase activity were significantly increased $(\mathrm{P}<0.05$ and $\mathrm{P}<0.01$, respectively). The current study indicated that the protective effects of ANE against bone loss were mediated, at least in part, via inhibition of the release of ROS and bone resorption. These results suggested that ANE could have therapeutic value in the treatment of osteoporosis.
\end{abstract}

Correspondence to: Dr Guankui Du or Dr Man Xiao, Department of Biochemistry and Molecular Biology, Hainan Medical College, 3 Xueyuan Road, Haikou, Hainan 571199, P.R. China

E-mail: dugk1983@hotmail.com

E-mail: xiaoman_shen2004@163.com

${ }^{*}$ Contributed equally

Key words: osteoporosis, areca nut extract, oxidative stress, receptor activator of nuclear factor- $\kappa \mathrm{B}$ ligand, osteoprotegerin

\section{Introduction}

Osteoporosis, which is a disease characterized by loss of bone mass and structural deterioration of the bone microarchitecture (1), has become a major health problem worldwide. Approximately $35 \%$ of women above the age of 65 suffer from primary osteoporosis (2). Osteoporosis leads to an increased risk of bone fragility and susceptibility to fracture (3). Estrogen deficiency results in rapid and marked loss of bone mineral content (4) and hormonal estradiol esters and conjugated estrogens have been used to prevent and treat osteoporosis in women. However, the risk/benefit ratio of prolonged treatment is accompanied by the undesired side-effects of increased risk of breast cancer, stroke and thromboembolism (5).

Betel quid, which is comprised of betel leaf, areca nut, and slaked lime, is the fourth most commonly used psychoactive substance worldwide, after tobacco, alcohol and caffeine (6). Betel quid chewing is reported to reduce stress, increase alertness and promote feelings of well-being (7-9). Areca nuts are rich in phenolics and have antioxidant properties (10). Betel quid has a high capacity to inhibit lipid peroxidation compared with vitamin E (6). Areca nut extract (ANE) has a high level of total phenolic content and displays antioxidant properties (11). Treatment of mice with ANE was previously demonstrated to reverse the oxidative damage induced in hepatic tissues by carbon tetrachloride (12). Furthermore, the areca nut extract CC-516, isolated by ethanol extraction, has been indicated to have effective anti-inflammatory/anti-melanogenesis properties (13). ANE has been reported to modulate nitric oxide generation via downregulation of inducible nitric oxide synthase levels and nuclear factor (NF)- $\kappa \mathrm{B}$ signaling both in vitro and in vivo (14).

Osteoprotegerin (OPG) is a member of the tumor necrosis factor (TNF) receptor superfamily. It functions as a decoy receptor by binding to the receptor activator of $N F-\kappa B$ ligand (RANKL) and competitively inhibiting the interaction between RANKL and RANK (15). The molecular mechanisms of RANK/RANKL/OPG interactions are critical to osteoclast differentiation and the regulatory system involved in bone homeostasis (16). RANKL is secreted by osteoblasts; following binding to RANK on the surface of preosteoclasts, RANKL stimulates the activation and development of osteoclasts. OPG is a powerful inhibitor of osteoclast formation 
and inhibits bone resorption (17). It has been reported that the RANKL/OPG ratio may determine the delicate balance between bone resorption and synthesis (18).

Micro-computed tomography (micro-CT) is a well-known imaging technique (19-21), which uses the absorption of $\mathrm{X}$-rays by sample material to create shadow images, which are used to generate cross-sectional images by the application of back-projection algorithms (22). Micro-CT has been used to characterize cancellous and cortical bone strain in an in vivo mouse tibial loading model (23), a mouse and human jaw joint (24) and the vascular bed in bone metastasis in a rat model (25).

It has previously been reported that estrogen deficiency causes bone loss by mediating oxidative stress-enhanced expression of cytokines that promote osteoclastic bone resorption (26). The aim of the present study was to investigate the protective effect of ANE on bone loss in ovariectomy (OVX)-induced osteoporosis mice. Following the administration of ANE, 3D micro-CT was used to study changes in the trochanteric region of the femur of OVX mice. The $\mathrm{OPG} / \mathrm{RANKL}$ and oxidative stress levels were also measured. These findings were used to evaluate the roles of RANKL, OPG and the RANKL/OPG ratio in OVX mice and the effects of ANE antioxidant activity on bone mineral density and levels of oxidative stress.

\section{Materials and methods}

Plant material and extraction. A total of $1 \mathrm{~kg}$ fresh tender nuts of Areca catechu were purchased directly from the grower (Herb Market; Haikou, China) in July 2013. Nuts (200 g) were chopped into cubes $\left(0.5-1 \mathrm{~cm}^{3}\right)$ using a blender and extracted using $500 \mathrm{ml}$ water at $4^{\circ} \mathrm{C}$ overnight. The supernatant was collected and concentrated by lyophilization at $-4^{\circ} \mathrm{C}$. The lyophilized powder was weighed, redissolved in $\mathrm{ddH}_{2} \mathrm{O}$ and stored at $-20^{\circ} \mathrm{C}$ before use. A solution of $3 \%$ ANE was prepared immediately before each experiment.

2,2-diphenyl-1-picrylhydrazyl (DPPH) assay. The ability of the prepared extracts to scavenge DPPH radicals was determined using the method described by Wang et al (27). Briefly, $10 \mu \mathrm{l} \mathrm{ANE}$ in different concentrations was added to $290 \mu \mathrm{l}$ methanol solution of DPPH (0.1 mM; Sigma-Aldrich; Merck Millipore, Darmstadt, Germany). The final concentrations of ANE were $0.5,1,2,4,8,15,20,25,30$ and $40 \mathrm{~g} / 1$. Vitamin C was employed as a reference. The concentrations of vitamin $\mathrm{C}$ were $0.5,1,2,4,8,15$ and $30 \mathrm{mg} / \mathrm{l}$. The solution was mixed and then left at room temperature for $30 \mathrm{~min}$ in the dark. The absorbance of the resulting solution was read at $519 \mathrm{~nm}$. The radical scavenging activity was calculated as a percentage of DPPH discoloration using the following equation: DPPH radical scavenging $(\%)=\left(\mathrm{A}_{\text {control }}-\mathrm{A}_{\text {sample }}\right) /\left(\mathrm{A}_{\text {control }} \mathrm{x} 100 \%\right)$. $\mathrm{A}_{\text {sample }}$ is the absorbance of the DPPH solution when the extract has been added at a particular level and $\mathrm{A}_{\text {control }}$ is the absorbance of the DPPH solution without the extract added. All analyses were run in triplicate. $\mathrm{IC}_{50}$ values denote the concentration of extract required to decrease the absorbance at $519 \mathrm{~nm}$ by $50 \%$.

Animals and treatment. A total of 24, 8-week-old female Kun Ming (KM) mice were obtained from Guangdong Medical
Laboratory Animal Center (Guangzhou, China). Mice (weight, 21-23 g) were housed in an environmentally controlled animal facility and maintained in a 12-h light/dark schedule at $23 \pm 2^{\circ} \mathrm{C}$ throughout the experimental period. Mice were fed a standard laboratory diet and given free access to deionized drinking water. The mice were randomly divided into three groups ( $\mathrm{n}=8$ per group): I, sham-operated control; II, bilateral OVX; III, bilateral OVX + ANE. This study was carried out in strict accordance with the recommendations in the Guide for the Care and Use of Laboratory Animals of the National Institutes of Health (Bethesda, MD, USA). The protocol was approved by the Committee on the Ethics of Animal Experiments of Hainan Medical College (Haikou, China; permit no. hy2013081601). All surgery was performed after intraperitoneal injection of $50 \mathrm{mg} / \mathrm{kg}$ of $1 \%$ sodium pentobarbital (Sigma-Aldrich; Merck Millipore) anesthesia, and all efforts were made to minimize suffering.

Under sodium pentobarbital anesthesia, bilateral (dorsolateral) ovariectomies were performed in Groups II and III, while sham operations were performed in Group I. After 7 days of recovery, Group III were treated orally with $3 \%$ aqueous ANE at a single dose of $300 \mathrm{mg} / \mathrm{kg}$ body weight $(\sim 0.2 \mathrm{ml}$ of $30 \mathrm{~g} / \mathrm{l}$ ANE, which exhibited an optimum antioxidant capacity to scavenge free radicals in the aforementioned DPPH assay) body weight daily for 6 months. The other two groups received deionized water as a placebo. During the period of ANE treatment, Group I was pair-fed with the experimental Groups II and III to minimize the impact of any altered food intake in the experimental groups.

At the end of the experiment, all animals were sacrificed (mice were treated with $3 \% \mathrm{CO}_{2}$ inhalation) and samples of whole blood were collected by cardiac puncture. Blood was allowed to clot for $30 \mathrm{~min}$ and serum was then separated by centrifugation at $1,500 \times \mathrm{g}$ for $10 \mathrm{~min}$. Soon after the blood collection, the femora were isolated, fixed in $10 \%$ formaldehyde and stored at $-20^{\circ} \mathrm{C}$.

Biochemical parameters. Serum levels of hydrogen peroxide $\left(\mathrm{H}_{2} \mathrm{O}_{2}\right)$, malondialdehyde (MDA), glutathione (GSH) and catalase (CAT) activity were measured using respective kits (Nanjing Jiancheng Bioengineering Institute, Nanjing, China), according to the manufacturer's instructions.

RANKL and $O P G$ analysis. Markers of bone turnover in the serum were measured using enzyme-linked immunosorbent assay (ELISA) kits for RANKL and OPG (R\&D Systems, Inc., Minneapolis, MN, USA). All ELISA procedures were performed according to the manufacturer's protocols.

Bone microarchitecture assessment by micro-CT. Bone microarchitecture in the distal femur was scanned using Explore Locus SP Pre-Clinical Specimen Micro-CT (GE Healthcare Bio-Sciences, Pittsburgh, PA, USA) with $8 \mathrm{~mm}$ resolution; tube voltage $80 \mathrm{kV}$ and tube current $80 \mu \mathrm{A}$. The reconstruction and $3 \mathrm{D}$ quantitative analyses were performed using the software (MicroView 1.1) provided with the micro-CT system (GE Healthcare Bio-Sciences). The same settings for scanning and analysis were used for all samples. In the femora, scanning regions were confined to the distal metaphysis, extending proximally $2.0 \mathrm{~mm}$ from the proximal tip of the primary spongiosa. 
The trabecular bone region was outlined for each micro-CT section. Within these regions, trabecular bone was separated from cortical bone with boundaries defined by the endocortical bone surfaces. The following 3D indices in the defined region of interest were analyzed: Proportion of bone volume in total volume (BV/TV), trabecular number (Tb.N), trabecular separation (Tb.Sp), trabecular thickness (Tb.Th), structure model index (SMI) and bone mineral density (BMD) (28). The operator conducting the scan analysis was blinded to the treatments associated with the specimens.

Statistical analysis. Data are presented as the mean \pm standard error of the mean. Data were analyzed by the Statistical Product and Service Solutions (SPSS) program version 16 (SPSS, Inc., Chicago, IL, USA). Group differences were determined using the Student's t-test. $\mathrm{P}<0.05$ was considered to indicate a statistically significant difference.

\section{Results}

Free radical scavenging activity of ANE. The soluble free radical DPPH is well known as a good hydrogen abstractor, yielding DPPH-H as a by-product (29). The antioxidant activity of ANE and vitamin C (a positive control) was measured based on the scavenging activities for stable DPPH radicals as presented in Fig. 1. DPPH scavenging activity was found to be $36.7 \%$ (8 g/l), 60.5\% (15 g/l), 67.2\% (20 g/l), 70.2\% (25 g/l), $72.3 \%(30 \mathrm{~g} / \mathrm{l})$ and $72.9 \%$ (40 g/l) for ANE. The half maximal $\mathrm{IC}_{50}$ values of $\mathrm{ANE}$ for the scavenging of DPPH radicals were $12,621.4 \mathrm{mg} / \mathrm{l}$ (ANE) and $5.3 \mathrm{mg} / \mathrm{ml}$ (vitamin C). These results suggested that $30 \mathrm{~g} / 1 \mathrm{ANE}$ exhibited an optimum antioxidant capacity to scavenge DPPH free radicals.

Body weight. The body weights of the animals in each group were measured monthly (Fig. 2). There were no significant differences in body weight between groups I, II and III.

Effect of ANE on the structural properties of bone. Alterations in the bone architecture of the femur is considered important in postmenopausal osteoporosis and is related to bone strength. To evaluate the effect of OVX and ANE on femur bone structure, architectural parameters of the femur were assessed in $3 \mathrm{D}$ micro-CT images (Fig. 3). In the OVX group (II), the BV/TV $(\mathrm{P}<0.05)$, Tb.Th $(\mathrm{P}<0.05)$, Tb.N $(\mathrm{P}<0.01)$ and BMD $(\mathrm{P}<0.01)$ were significantly lower as compared with the sham-operated control group $(\mathrm{I})$, Tb.Sp $(\mathrm{P}<0.01)$ and SMI $(\mathrm{P}<0.05)$ were significantly higher (Fig. 4). In the ANE treatment group (III), BV/TV, Tb.Th, Tb.N and BMD were significantly increased compared with the OVX group (II) (all $\mathrm{P}<0.05$ ), while Tb.Sp and SMI were significantly decreased (both $\mathrm{P}<0.05$ ).

Effect of ANE on serum RANKL, OPG and RANKL/OPG ratio in $O V X$ mice. In the present study, it was hypothesized that ANE inhibited the bone resorption process. OPG and RANKL levels are critical for osteoclast regulation (30); therefore, serum levels of OPG and RANKL were determined by ELISA. In the OVX group (II), serum levels of OPG were significantly decreased compared with the sham-operated control group (I), whereas RANKL levels were significantly increased (both $\mathrm{P}<0.05$; Fig. $5 \mathrm{~A}$ and $\mathrm{B})$. The RANKL/OPG ratio was also

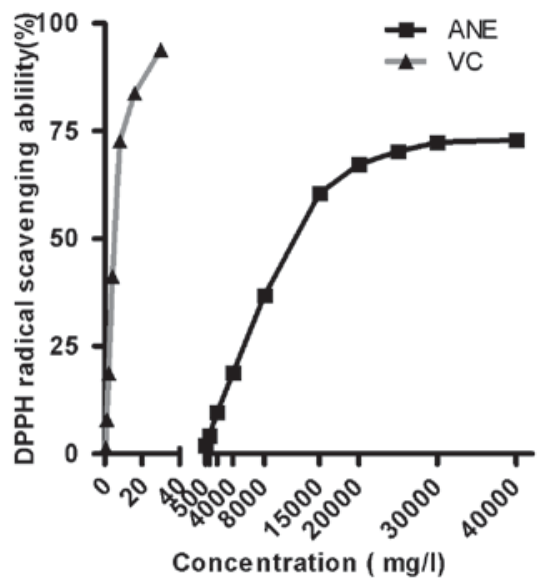

Figure 1. DPPH assay to determine optimum ANE concentration for free radical scavenging activity. ANE, areca nut extract; VC, vitamin C; DPPH, 2,2-diphenyl-1-picrylhydrazyl.

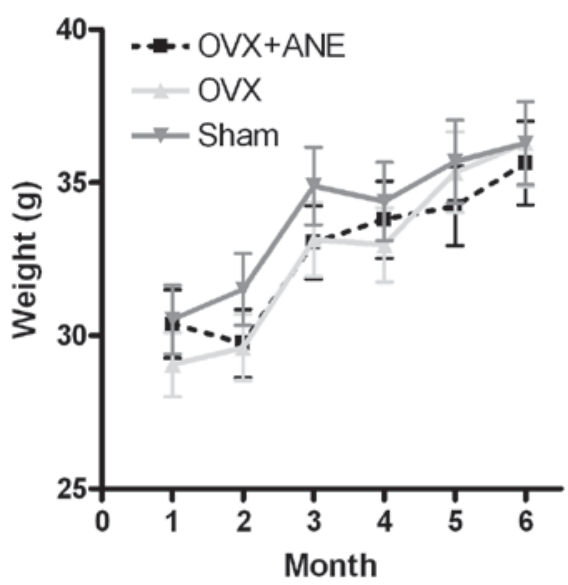

Figure 2. Effect of ANE treatment on body weight in OVX mice ( $\mathrm{n}=8$ in each group). Body weights were measured once a month. OVX, ovariectomy; ANE, areca nut extract.

significantly increased in the OVX group (II) compared with the sham-operated control group (I) $(\mathrm{P}<0.05$; Fig. 5C). ANE treatment significantly reversed the OVX-induced decrease in serum RANKL levels and the increase in serum OPG levels compared with the OVX non-treated group (II) (both $\mathrm{P}<0.05$; Fig. 5A and B). Therefore, the RANKL/OPG ratio was significantly decreased by ANE treatment compared with the OVX non-treated group (II) $(\mathrm{P}<0.05$; Fig. 5C).

Evaluation of oxidative stress. Estrogen deficiency in the OVX group (I) induced changes in oxidative stress parameters, with significantly increased levels of $\mathrm{H}_{2} \mathrm{O}_{2}$ and MDA in the serum compared with the sham-operated control group (both $\mathrm{P}<0.05$; Fig. 6A and B). Furthermore, CAT activity (a biomarker for antioxidant activity) in the serum was significantly decreased in the OVX group compared with the sham-operated group (I) six months after surgery $(\mathrm{P}<0.01$; Fig. $6 \mathrm{C})$. Treatment with ANE for 6 months significantly decreased both $\mathrm{H}_{2} \mathrm{O}_{2}$ and 

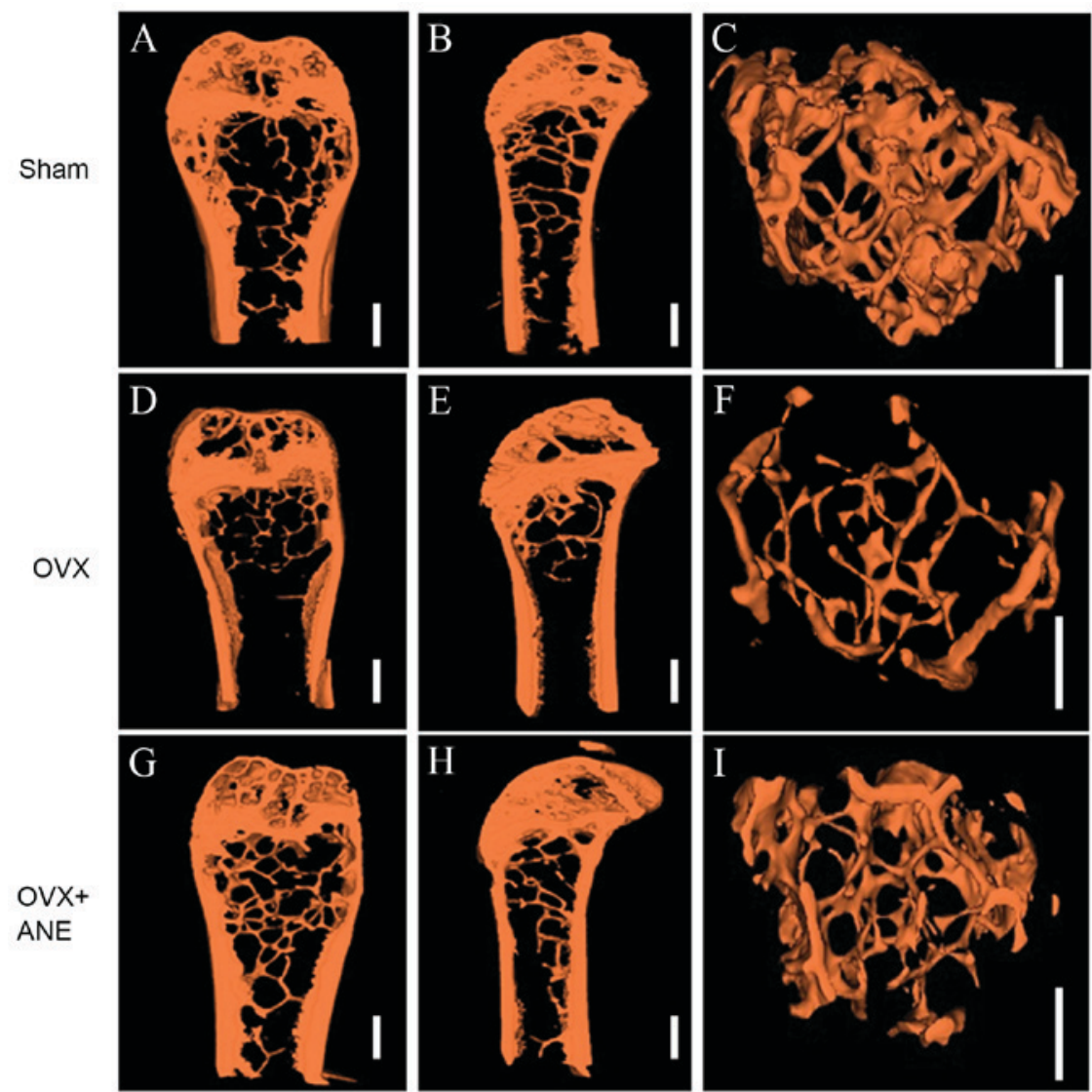

Figure 3. Three-dimensional micro-computed tomography images ( $8 \mathrm{~mm}$ resolution) of coronal (A, D, G), sagittal (B, E, H) and trabecular (C, F, I) fractures of the femoral condyle. OVX, ovariectomy; ANE, areca nut extract.
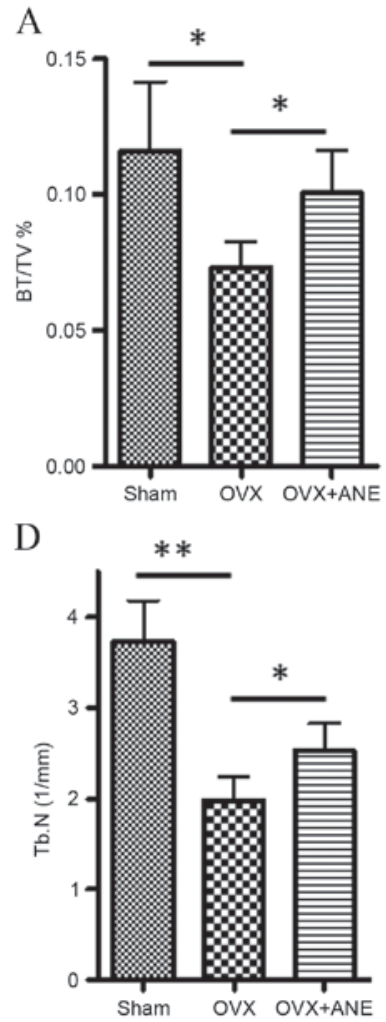

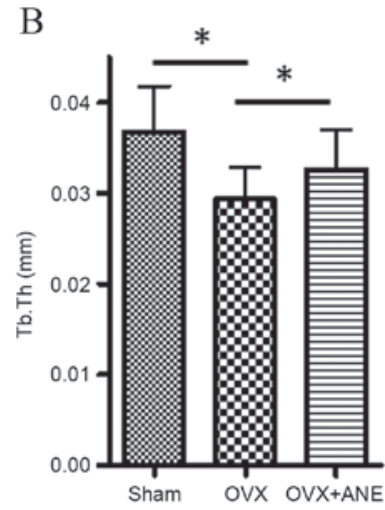

$\mathrm{E}$

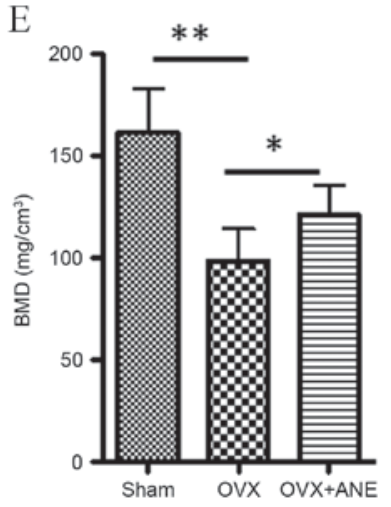

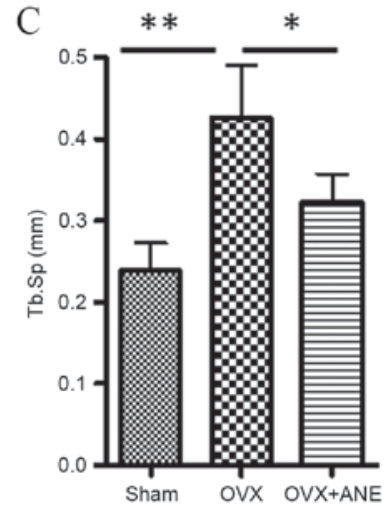

$\mathrm{F}$

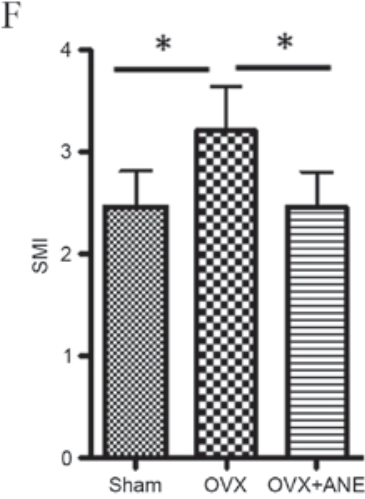

Figure 4. Cortical bone parameters in OVX mice, evaluated by 3D micro-computed tomography analysis (n=8 in each group). (A) BV/TV, (B) Tb.Th, (C) Tb.Sp, (D) Tb.N, (E) BMD and (F) SMI. ${ }^{*} \mathrm{P}<0.05$, ${ }^{* *} \mathrm{P}<0.01$. OVX, ovariectomy; ANE, areca nut extract; BV/TV, proportion of bone volume in total volume (bone volume density); Tb.Th, trabecular thickness; Tb.Sp, trabecular separation; Tb.N, trabecular number; BMD, bone mineral density; SMI, structure model index. 

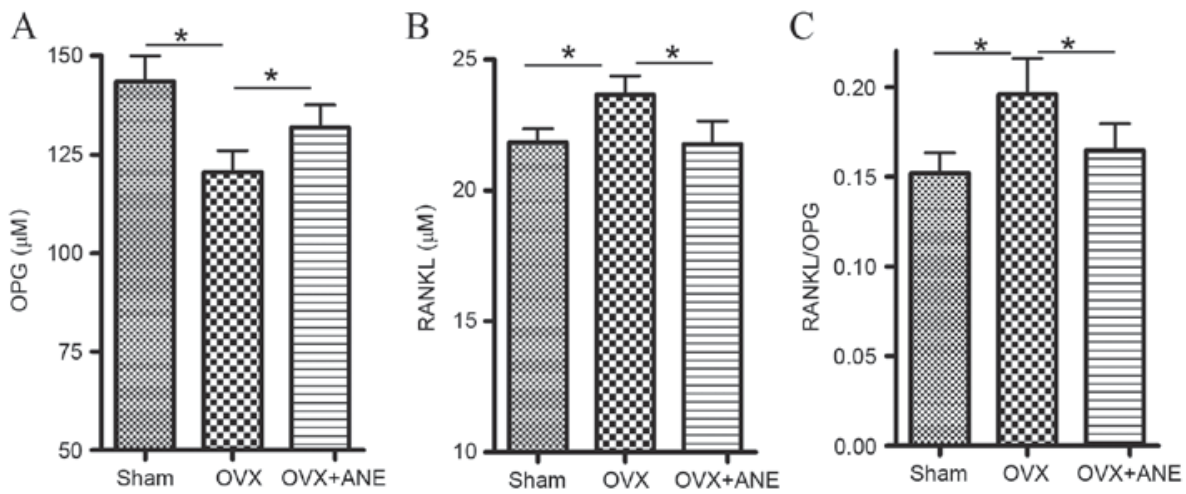

Figure 5. Effect of ANE on serum levels of OPG, RANKL and RANKL/OPG ratio in OVX mice ( $\mathrm{n}=8$ in each group). Serum levels of (A) OPG and (B) RANKL were determined using murine OPG and RANKL ELISA assays and (C) the RANKL/OPG ratio was calculated. "P<0.05. OVX, ovariectomy; ANE, areca nut extract; OPG, osteoprotegerin; RANKL, receptor activator of nuclear factor-kB ligand.
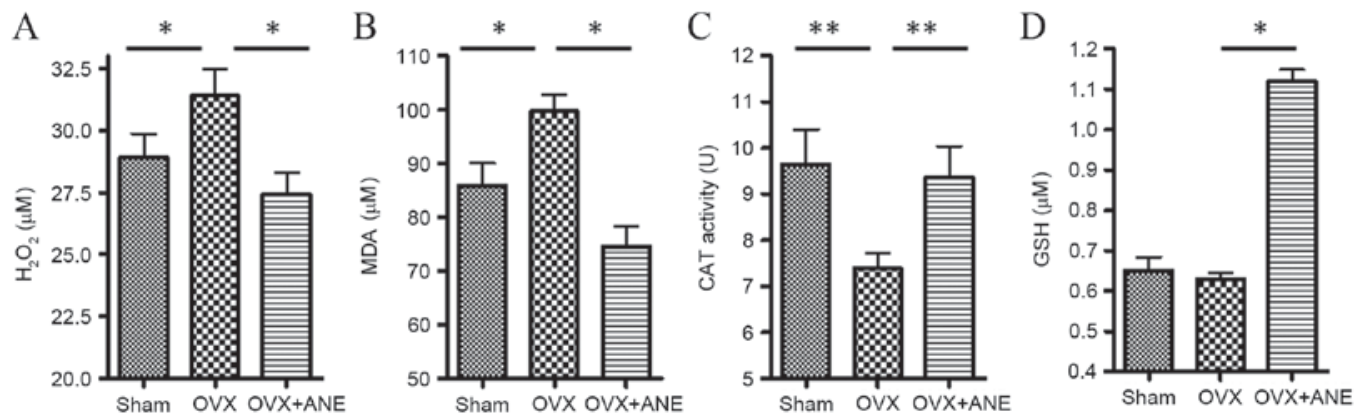

Figure 6. Effect of ANE on oxidative stress in OVX mice ( $\mathrm{n}=8$ in each group). Serum levels of (A) $\mathrm{H}_{2} \mathrm{O}_{2}$, (B) MDA, (C) GSH and (D) CAT activity were measured. " $\mathrm{P}<0.05$, ${ }^{* *} \mathrm{P}<0.01$. OVX, ovariectomy; ANE, areca nut extract; $\mathrm{H}_{2} \mathrm{O}_{2}$, hydrogen peroxide; MDA, malondialdehyde; GSH, glutathione; CAT, catalase.

MDA levels in the serum (both $\mathrm{P}<0.05$; Fig. 6A and B) and significantly increased serum CAT activity as compared with the OVX non-treated group (II) $(\mathrm{P}<0.01 ; \mathrm{Fig}$. 6C). In addition, ANE treatment significantly elevated serum GSH levels after 6 months of treatment compared with the OVX non-treated group (II) $(\mathrm{P}<0.05$; Fig. 6D).

\section{Discussion}

The purpose of the present study was to evaluate the effects of ANE on bone metabolism in OVX female rats. This was investigated by measuring bone density and biochemical parameters, including the serum levels of OPG and RANKL, as well as changes in the levels of oxidative stress markers such as $\mathrm{H}_{2} \mathrm{O}_{2}$, MDA, CAT activity and GSH.

ROS are produced by the mitochondrial electron transport chain or other enzymatic systems, such as nicotinamide adenine dinucleotide phosphate (NADPH) oxidases, cytochrome P-450, cyclo-oxygenase, aldehyde oxidase, dihydroorotate dehydrogenase, tryptophan dioxygenase, nitric oxide synthase and xanthine oxidase (31). Traditionally, mitochondria are considered to be the main source of ROS overproduction. However, excessive stimulation of NADPH can also result in oxidative stress (32), which induces an imbalance between pro-oxidants and antioxidants in cells and tissues.

Oxidative stress is related to aging and bone biology (33). Estrogen deficiency increases the generation of ROS, and the adverse effects of estrogen loss on bone may be attenuated by antioxidants (34). OVX leads to significant bone loss in the femora and vertebrae of wild type mice with comparable osteogenic activity (35). In the present study, OVX was associated with increased serum levels of $\mathrm{H}_{2} \mathrm{O}_{2}$ and MDA and decreased CAT activity. These results provided evidence in support of the theory that estrogen deficiency results in increased ROS generation.

The beneficial effects of ANE may be mediated by protecting against oxidative stress. ANE was previously found to be rich in phenolic compounds, which possess an ideal structural chemistry for free radical scavenging activity (10). Sazwi et al (11) reported that ANE exhibited strong cytoprotective effects, comparable to those of ascorbic acid. Previous results have also shown that ANE has higher antioxidant activity compared with $\alpha$-tocopherol (36). Cui et al (37) reported that extracts containing polyphenolic compounds showed extremely potent cytoprotective effects against $\mathrm{H}_{2} \mathrm{O}_{2}$-induced damage. The current results indicated that ANE treatment inhibited the OVX-induced increase in both $\mathrm{H}_{2} \mathrm{O}_{2}$ and MDA levels, and reversed the OVX-induced decrease in CAT activity. The GSH level was also significantly elevated in OVX mice treated with ANE. These results suggested that ANE had an antioxidant effect in OVX-induced osteoporosis mice.

The balance of bone formation and resorption is mediated by osteoblasts (bone-forming cells) and osteoclasts (bone resorption cells), respectively (38). ROS are involved in apoptosis of osteoblasts and osteocytes and osteoclastogenesis and, 
therefore, also in the process of bone resorption (39). ROS can also suppress the differentiation of osteoblasts (40). Dysregulation of bone homeostasis leads to bone diseases (41). In the current study, OVX induced oxidative stress along with the induction of osteoporosis in mice. Measurements of microarchitectural parameters such as BV/TV, Tb.N, Tb.Sp, Tb.Th, SMI and BMD can be used to estimate bone strength (42). BV/TV, Tb.Th, Tb.N and BMD were significantly decreased and Tb.Sp and SMI were significantly increased in the KM mice 6 months after bilateral OVX. These effects were partially reversed by ANE treatment for 6 months, which improved the bone structural indices, BMD and trabecular thickness that had been compromised by OVX.

The molecular triad of OPG/RANK/RANKL plays a crucial role in bone remodeling (43). OPG/RANK/RANKL functions as a pivotal molecular link between osteoblast and osteoclast activity. RANKL activates mature osteoclasts and mediates osteoclastogenesis, while OPG acts a decoy receptor for RANKL, preventing it from binding to and activating RANK (44). In a previous study, exogenous superoxide radicals or $\mathrm{H}_{2} \mathrm{O}_{2}$ were shown to stimulate RANKL and TNF- $\alpha$ expression in osteoblasts in vitro, suggesting that ROS activate osteoclastic bone resorption via RANKL derived from osteoblasts (34). Superoxide dismutase 1 deficiency increases ROS levels and induces cellular abnormalities in osteoblasts (45). Estrogen deficiency increases the generation of ROS, and the adverse effects of estrogen deficiency on bone can be prevented by antioxidants (34). The current study demonstrated that, compared with the sham-operated group, OVX mice had increased levels of RANKL and decreased levels of OPG, with a consequent increase in the RANKL/OPG ratio. ANE treatment was indicated to inhibit these effects; in the $\mathrm{OVX}+\mathrm{ANE}$ group, the RANKL/OPG ratio was significantly lower compared with the OVX group. These results suggest that an alteration in RANKL/OPG may be involved in the antioxidant capacity of ANE.

In summary, the current study suggests that antioxidant properties of ANE alleviate oxidative stress in OVX-induced osteoporosis mice. Treatment with ANE could affect the serum levels of OPG and RANKL, decreasing the RANKL/OPG ratio. Therefore, ANE is indicated to have a protective effect against OVX-induced osteoporosis through regulating oxidant homeostasis.

\section{Acknowledgements}

The authors thank the Guangdong Medical Laboratory Animal Center for supplying mice. This study was funded by Natural Science Foundation of Hainan Province (grant no. 812173 to Dr Chen Rong) and Innovative Experiment Projects (grant no. 201211810052).

\section{References}

1. Kanis JA, Melton LJ III, Christiansen C, Johnston CC and Khaltaev N: The diagnosis of osteoporosis . J Bone Miner Res 9: 1137-1141, 1994.

2. Melton LJ III, Chrischilles EA, Cooper C, Lane AW and Riggs BL: How many women have osteoporosis? JBMR Anniversary Classic. JBMR, Volume 7, Number 9, 1992. J Bone Miner Res 20: 886-892, 2005.
3. McNamara LM: Perspective on post-menopausal osteoporosis: Establishing an interdisciplinary understanding of the sequence of events from the molecular level to whole bone fractures. J R Soc Interface 7: 353-372, 2010.

4. Sliwiński L, Folwarczna J, Janiec W, Grynkiewicz G and Kuzyk K: Differential effects of genistein, estradiol and raloxifene on rat osteoclasts in vitro. Pharmacol Rep 57: 352-359, 2005.

5. Moreira AC, Silva AM, Santos MS and Sardão VA: Phytoestrogens as alternative hormone replacement therapy in menopause: What is real, what is unknown. J Steroid Biochem Mol Biol 143: 61-71, 2014

6. Sullivan RJ, Allen JS, Otto C, Tiobech J and Nero K: Effects of chewing betel nut (Areca catechu) on the symptoms of people with schizophrenia in Palau, Micronesia. Br J Psychiatry 177: 174-178, 2000.

7. Bhandare AM, Kshirsagar AD, Vyawahare NS, Hadambar AA and Thorve VS: Potential analgesic, anti-inflammatory and antioxidant activities of hydroalcoholic extract of Areca catechu L. nut. Food Chem Toxicol 48: 3412-3417, 2010.

8. Azeez S, Amudhan S: Adiga S, Vasanthakumar A, Rao N, Udupa LA and Sanath H: Wound healing profile of Areca catechu extracts on different wound models in wistar rats. Kuwait Med J 13: 48-52, 2007.

9. Dar A and Khatoon S: Behavioral and biochemical studies of dichloromethane fraction from the areca catechu nut. Pharmacol Biochemistry Behavior 65: 1-6, 2000.

10. Li CC and Lin ES: Antiradical capacity and reducing power of different extraction method of Areca catechu seed. African J Biotechnol 9: 7831-7836, 2010.

11. Sazwi NN, Nalina T and Abdul Rahim ZH: Antioxidant and cytoprotective activities of Piper betle, Areca catechu, Uncaria gambir and betel quid with and without calcium hydroxide. BMC Complement Altern Med 13: 351, 2013.

12. Pithayanukul P, Nithitanakool S and Bavovada R: Hepatoprotective potential of extracts from seeds of Areca catechu and nutgalls of Quercus infectoria. Molecules 14: 4987-5000, 2009.

13. Lee KK and Choi JD: The effects of areca catechu L extract on anti-inflammation and anti-melanogenesis. Int J Cosmet Sci 21: 275-284, 1999.

14. Lee KP, Sudjarwo GW, Kim JS, Dirgantara S, Maeng WJ and Hong $\mathrm{H}$ : The anti-inflammatory effect of Indonesian Areca catechu leaf extract in vitro and in vivo. Nutr Res Pract 8: 267-271, 2014.

15. Hofbauer LC, Khosla S, Dunstan CR, Lacey DL, Boyle WJ and Riggs BL: The roles of osteoprotegerin and osteoprotegerin ligand in the paracrine regulation of bone resorption. J Bone Miner Res 15: 2-12, 2000.

16. Lorenzo J, Horowitz $M$ and Choi Y: Osteoimmunology: Interactions of the bone and immune system. Endocr Rev 29: 403-440, 2008.

17. Simonet WS, Lacey DL, Dunstan CR, Kelley M, Chang MS, Lüthy R, Nguyen HQ, Wooden S, Bennett L, Boone T, et al: Osteoprotegerin: A novel secreted protein involved in the regulation of bone density. Cell 89: 309-319, 1997.

18. Karst M, Gorny G, Galvin RJ and Oursler MJ: Roles of stromal cell RANKL, OPG, and M-CSF expression in biphasic TGF-beta regulation of osteoclast differentiation. J Cell Physiol 200: 99-106, 2004.

19. Masschaele B, Cnudde V, Dierick M, Jacobs P, Van Hoorebeke L and Vlassenbroeck J: UGCT: New X-ray radiography and tomography facility. Nuclear Instruments Methods Physics Res Section 580: 266-269, 2007.

20. Welkenhuyzen F, Kiekens K, Pierlet M, Dewulf W, Bleys P, Kruth JP and Voet A: Industrial computer tomography for dimensional metrology: Overview of influence factors and improvement strategies, In: Proceedings of the 4th International Conference on Optical Measurement Techniques for Structures and Systems: Optimess2009, Antwerp, pp401-410, 2009.

21. Salih WH, Buytaert JA, Aerts JR, Vanderniepen P, Dierick M and Dirckx JJ: Open access high-resolution 3D morphology models of cat, gerbil, rabbit, rat and human ossicular chains. Hear Res 284: 1-5, 2012.

22. Dierick M, Masschaele B and Van Hoorebeke L: Octopus, a fast and user-friendly tomographic reconstruction package developed in LabView ${ }^{\circledR}$. Meas Sci Technol 15: 1366-1370, 2004.

23. Yang H, Butz KD, Duffy D, Niebur GL, Nauman EA and Main RP: Characterization of cancellous and cortical bone strain in the in vivo mouse tibial loading model using microCT-based finite element analysis. Bone 66: 131-139, 2014. 
24. Renders GA, Mulder L, Lin AS, Langenbach GE, Koolstra JH Guldberg RE and Everts V: Contrast-enhanced microCT EPIC- $\mu \mathrm{uCT}$ ex vivo applied to the mouse and human jaw joint. Dentomaxillofac Radiol 43: 20130098, 2014

25. Nyangoga H, Mercier P, Libouban H, Baslé MF and Chappard D: Three-dimensional characterization of the vascular bed in bone metastasis of the rat by microcomputed tomography MicroCT. PLoS One 6: e17336, 2011.

26. Lean JM, Davies JT, Fuller K, Jagger CJ, Kirstein B, Partington GA, Urry ZL and Chambers TJ: A crucial role for thiol antioxidants in estrogen-deficiency bone loss. J Clin Invest 112: 915-923, 2003.

27. Wang CZ, Yuan HH, Bao XL and Lan MB: In vitro antioxidant and cytotoxic properties of ethanol extract of Alpinia oxyphylla fruits. Pharm Biol 51: 1419-1425, 2013.

28. Majumdar S, Genant HK, Grampp S, Newitt DC, Truong VH, Lin JC and Mathur A: Correlation of trabecular bone structure with age, bone mineral density, and osteoporotic status: In vivo studies in the distal radius using high resolution magnetic resonance imaging. J Bone Miner Res 12: 111-118, 1997.

29. du Toit R, Volsteedt Y and Apostolides Z: Comparison of the antioxidant content of fruits, vegetables and teas measured as vitamin C equivalents. Toxicology 166: 63-69, 2001.

30. Khosla S: Minireview: The OPG/RANKL/RANK system. Endocrinology 142: 5050-5055, 2001.

31. Apel K and Hirt H: Reactive oxygen species: Metabolism, oxidative stress, and signal transduction. Annu Rev Plant Biol 55: 373-399, 2004.

32. Finaud J, Lac G and Filaire E: Oxidative stress: Relationship with exercise and training. Sports Med 36: 327-358, 2006.

33. Bai XC, Lu D, Liu AL, Zhang ZM, Li XM, Zou ZP, Zeng WS, Cheng BL and Luo SQ: Reactive oxygen species stimulates receptor activator of NF-kappaB ligand expression in osteoblast. J Biol Chem 280: 17497-17506, 2005.

34. Manolagas SC: From estrogen-centric to aging and oxidative stress: A revised perspective of the pathogenesis of osteoporosis. Endocr Rev 31: 266-300, 2010.
35. Akune T, Ohba S, Kamekura S, Yamaguchi M, Chung UI, Kubota N, Terauchi Y, Harada Y, Azuma Y, Nakamura K, et al: PPARgamma insufficiency enhances osteogenesis through osteoblast formation from bone marrow progenitors. J Clin Invest 113: 846-855, 2004.

36. Huda-Faujan N, Noriham A, Norrakiah AS and Babji AS: Antioxidant activity of plants methanolic extracts containing phenolic compounds. African J Biotechnol 8: 484-489, 2009.

37. Cui Y, Kim DS and Park KC: Antioxidant effect of Inonotus obliquus. J Ethnopharmacol 96: 79-85, 2005.

38. Altindag O, Erel O, Soran N, Celik H and Selek S: Total oxidative/anti-oxidative status and relation to bone mineral density in osteoporosis. Rheumatol Int 28: 317-321, 2008

39. Almeida M, Han L, Martin-Millan M, O'Brien CA and Manolagas SC: Oxidative stress antagonizes Wnt signaling in osteoblast precursors by diverting beta-catenin from $\mathrm{T}$ cell factor-to forkhead box O-mediated transcription. J Biol Chem 282: 27298-27305, 2007.

40. Bai XC, Lu D, Bai J, Zheng H, Ke ZY, Li XM and Luo SQ: Oxidative stress inhibits osteoblastic differentiation of bone cells by ERK and NF-kappaB. Biochem Biophys Res Commun 314: 197-207, 2004.

41. Banfi G, Iorio EL and Corsi MM: Oxidative stress, free radicals and bone remodeling. Clin Chem Lab Med 46: 1550-1555, 2008.

42. Kazakia GJ and Majumdar S: New imaging technologies in the diagnosis of osteoporosis. Rev Endocr Metab Disord 7: 67-74, 2006.

43. Theoleyre S, Wittrant Y, Tat SK, Fortun Y, Redini F and Heymann D: The molecular triad OPG/RANK/RANKL: Involvement in the orchestration of pathophysiological bone remodeling. Cytokine Growth Factor Rev 15: 457-475, 2004.

44. Boyle WJ, Simonet WS and Lacey DL: Osteoclast differentiation and activation. Nature 423: 337-342, 2003.

45. Nojiri H, Saita Y, Morikawa D, Kobayashi K, Tsuda C, Miyazaki T, Saito M, Marumo K, Yonezawa I, Kaneko K, et al: Cytoplasmic superoxide causes bone fragility owing to low-turnover osteoporosis and impaired collagen cross-linking. J Bone Miner Res 26: 2682-2694, 2011. 